\title{
Deep learning method for Martian atmosphere reconstruction
}

\author{
Davide Amato *, Jay W. McMahon ${ }^{\dagger}$ \\ University of Colorado, Boulder, Colorado, 80303
}

The reconstruction of atmospheric properties encountered during Mars entry trajectories is a crucial element of post-flight mission analysis. We propose a deep learning architecture using a Long Short-Term Memory Network (LSTM) for the reconstruction of Martian density and wind profiles from inertial measurements and guidance commands. The LSTM is trained on a large set of Mars entry trajectories controlled through the Fully Numerical Predictor-corrector Entry Guidance (FNPEG) algorithm, with density and wind from the Mars Global Reference Atmospheric Model (GRAM) 2010. We examine the training of the network, ensuring that the LSTM generalizes well to samples not present in the training set, and assess the performance of the network on a separate training set. The errors of the reconstructed density and wind profiles are respectively within $0.54 \%$ and $1.9 \%$. Larger wind errors take place at high altitudes due to the decreased sensitivity of the trajectory in regions of low dynamic pressure. The LSTM architecture reliably reproduces the atmospheric density and wind encountered during descent.

\section{Nomenclature}

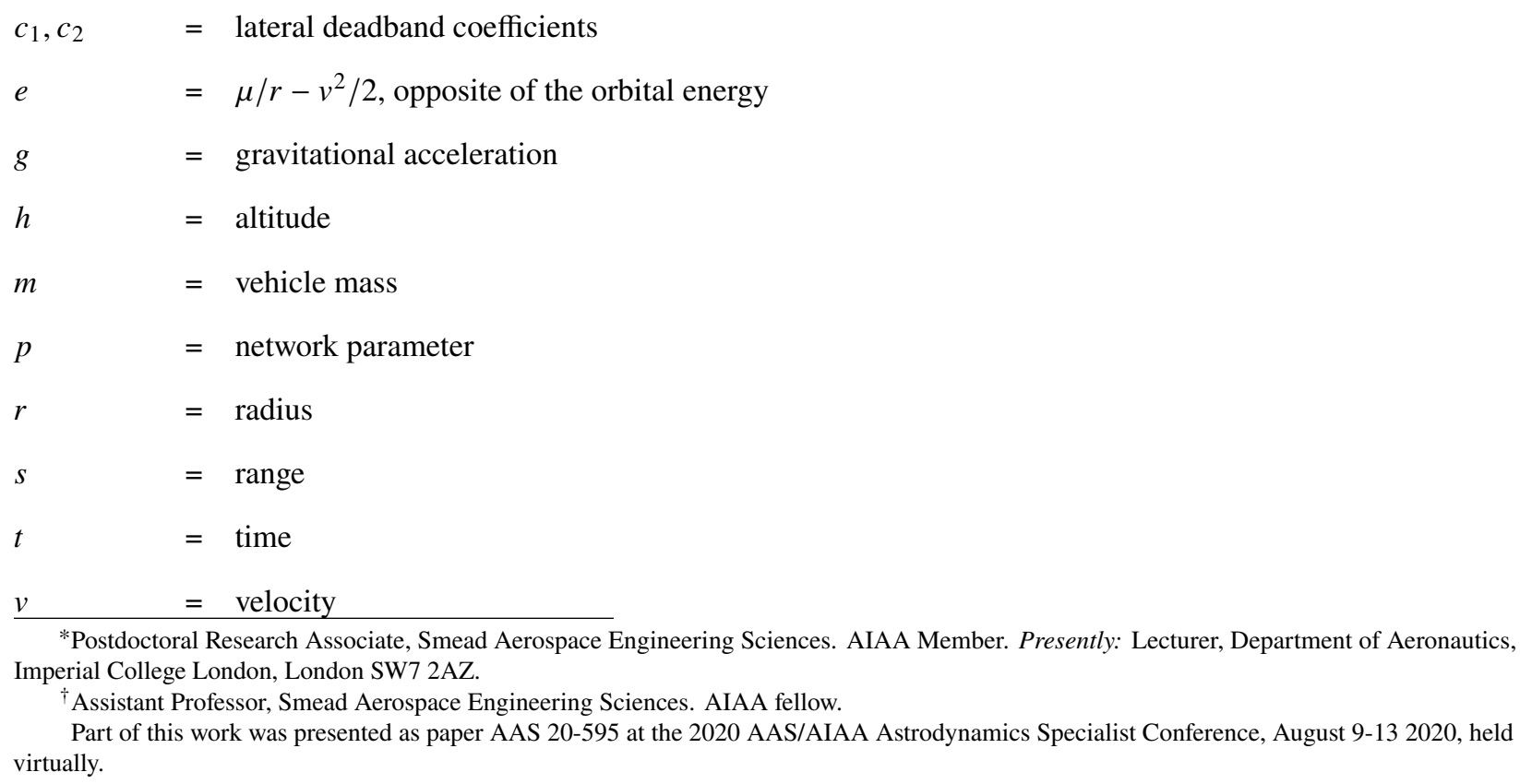

*Postdoctoral Research Associate, Smead Aerospace Engineering Sciences. AIAA Member. Presently: Lecturer, Department of Aeronautics, Imperial College London, London SW7 2AZ.

$\dagger$ Assistant Professor, Smead Aerospace Engineering Sciences. AIAA fellow.

Part of this work was presented as paper AAS 20-595 at the 2020 AAS/AIAA Astrodynamics Specialist Conference, August 9-13 2020, held virtually. 


\begin{tabular}{|c|c|c|}
\hline$w$ & $=$ & wind velocity magnitude \\
\hline$z$ & $=$ & range error \\
\hline$A$ & $=$ & reference area \\
\hline$A_{0}$ & $=$ & mean value of longitude-dependent density wave \\
\hline$B$ & $=$ & pseudo-density scaling factor \\
\hline$C$ & $=$ & dataset \\
\hline$D$ & $=$ & drag acceleration \\
\hline$L$ & $=$ & lift acceleration \\
\hline$P$ & $=$ & set of network parameters \\
\hline$S$ & $=$ & scale parameter of longitude-dependent density wave \\
\hline$U, V, W$ & $=$ & weight matrices \\
\hline$b, c$ & $=$ & bias vectors \\
\hline$f$ & $=$ & output of the LSTM forget layer \\
\hline$g$ & $=$ & output of the LSTM input layer \\
\hline $\boldsymbol{h}$ & $=$ & hidden state \\
\hline$i$ & $=$ & layer input \\
\hline $\boldsymbol{o}$ & $=$ & layer output \\
\hline $\boldsymbol{r}$ & $=$ & radius vector \\
\hline$s$ & $=$ & LSTM cell state \\
\hline $\boldsymbol{u}$ & $=$ & output of the LSTM output layer \\
\hline$v$ & $=$ & $\dot{\boldsymbol{r}}$, velocity in the Mars-fixed reference frame \\
\hline $\boldsymbol{v}_{\infty}$ & $=$ & $\boldsymbol{v}-\boldsymbol{w}$, velocity with respect to the flow \\
\hline$w$ & $=$ & wind velocity vector \\
\hline$x$ & $=$ & feature vector \\
\hline$y$ & $=$ & target vector \\
\hline $\boldsymbol{F}$ & $=$ & mapping from features to targets \\
\hline$L$ & $=$ & lift vector \\
\hline $\boldsymbol{D}$ & $=$ & drag vector \\
\hline$\alpha$ & $=$ & learning rate \\
\hline$\beta$ & $=$ & step-size regulation factor in the Gauss-Newton method \\
\hline$\Delta h$ & $=$ & altitude discretization interval \\
\hline$\Delta \Psi$ & $=$ & heading offset \\
\hline
\end{tabular}




$$
\begin{aligned}
& \gamma \quad=\text { flight-path angle } \\
& \eta \quad=\text { pseudo-density } \\
& \theta \quad=\text { latitude } \\
& \lambda \quad=\text { regularization weight } \\
& \mu \quad=\text { Mars gravitational parameter } \\
& \psi \quad=\text { heading angle } \\
& \rho \quad=\text { atmospheric density } \\
& \sigma \quad=\text { bank angle } \\
& \theta \quad=\text { longitude } \\
& \tau_{\text {dust }}=\text { background dust optical depth } \\
& \Omega \quad=\text { Mars angular velocity } \\
& \boldsymbol{\theta}=\text { activation function } \\
& \mathbf{\Xi}=\text { sequence of feature vectors } \\
& \xi=\text { unnormalized feature vector } \\
& \mathbf{\Omega}=\text { Mars angular velocity vector } \\
& \mathcal{L} \quad=\text { loss function } \\
& a_{x}, a_{y}, a_{z}=\text { measured acceleration components in the Mars-fixed frame } \\
& r_{x}, r_{y}, r_{z}=\text { position components in the Mars-fixed frame } \\
& v_{E}, v_{N}, v_{R}=\text { velocity components in the East, North, Radial frame } \\
& v_{x}, v_{y}, v_{z}=\text { velocity components in the Mars-fixed frame } \\
& C_{D} \quad=\text { drag coefficient } \\
& C_{L} \quad=\text { lift coefficient } \\
& \bullet \quad=\text { current } \\
& \bullet_{i} \quad=i \text { th timestep } \\
& \bullet_{E} \quad=\text { East } \\
& \bullet \quad=\text { North } \\
& \bullet_{\mathrm{i}} \quad=\text { initial } \\
& \bullet_{\mathrm{f}} \quad=\text { final } \\
& \bullet_{\mathrm{EI}} \quad=\text { Entry Interface }
\end{aligned}
$$

Superscripts

○ $=$ predicted 


\begin{tabular}{|c|c|}
\hline • & $=$ normalized \\
\hline$\bullet$ & $=$ mean \\
\hline • & $=$ standard deviation \\
\hline$j_{\bullet}$ & $=j$ th component of the feature or target vectors \\
\hline$\bullet$ & $=k$ th sample \\
\hline • $(l)$ & $=l$ th layer \\
\hline$\bullet *$ & $=$ guidance target \\
\hline
\end{tabular}

\section{Introduction}

$\mathrm{T}$ HE reconstruction of the atmospheric density and wind encountered during the Entry, Descent, and Landing (EDL) of a spacecraft at Mars is an essential component of post-flight analysis and performance evaluation. Estimating the atmospheric density and winds encountered during descent enables a more accurate assessment of the vehicle performance, besides providing information on Martian atmospheric processes.

Several works in the literature are concerned with the a posteriori reconstruction of the Martian atmosphere. Atmospheric density and temperature were derived from the Mars Pathfinder deceleration profile, with aerodynamic coefficients estimated from a preflight aerodynamic database [1]. A similar approach was employed during the aerobraking operations of the Mars Reconnaissance Orbiter (MRO), in which the density was recovered with high accuracy from accelerometer data [2]. Reconstructed density profiles for the descent of the MER rovers and the Phoenix lander are provided in Reference 3. In each case, the density experienced during the entry was lower than that predicted by Mars atmospheric models, which lead to downrange landing errors and highlights the importance of accurate density estimates. More recently, Dutta and others explicitly took into account state and process noise by estimating density through Extended and Unscented Kalman filters. In addition, uncertainties on aerodynamic coefficients were separated from those on atmospheric quantities by employing air data measurements available during the descent of the Mars Science Laboratory (MSL) [4, 5]. A comprehensive study and reconstruction of the density and wind experienced during the MSL descent was carried out with the aid of several different datasets, including remote sensing data from the Mars Reconnaissance Orbiter, air data from the Mars Entry Atmosphere Data System onboard the MSL entry vehicle, and post-flight trajectory data [6]. Notably, we are aware of no other works in which the wind profiles have been reconstructed along with the density. Wind uncertainties have played an important role in the selection of landing sites for past missions, especially for regions of the Mars surface where significant wind shear is expected [7].

This work is driven by the observation that the reconstruction of density and wind from inertial measurements relies on the solution of an inverse problem, in which the parameters of a dynamical system must be estimated from its trajectory. Deep learning algorithms based on neural networks have recently been shown to be very powerful for this 
task [8]. Neural networks are computational models defined by the sequential composition of nonlinear functions and affine transformations. Desired outputs can be obtained by appropriately choosing a set of parameters of the affine transformations. In supervised learning, the parameters are found through a numerical optimization process (the network training) minimizing a prescribed loss function on a given set of feature and target pairs [9]. From an engineering perspective, neural networks can be regarded as function approximators mapping a set of features into targets; the particular realization of the mapping being defined by the samples in the training set. Universal approximation theorems guarantee that neural networks can approximate a large class of functions [10, 11]. Notably, shallow neural networks have also been proposed for the onboard prediction of atmospheric quantities in entry trajectories and for autonomous spacecraft navigation $[12,13]$. A particular class of deep learning algorithms, the Recurrent Neural Networks (RNNs), has found extensive application in the processing of sequential data. However, the performance of classical RNNs generally degrades with increasing input sequence lengths due to gradients either vanishing or diverging during the network training. The Long Short-Term Memory (LSTM) memory RNN architecture successfully solved the problem of long-term dependencies by introducing mechanisms for the preservation of the gradient over long sequences [14].

In this work, we propose a deep learning method based on a LSTM network for the reconstruction of density and wind from EDL trajectories. The dataset for the training and testing of the network is generated by simulating a large number of entry trajectories obtained with different realizations of the Mars Global Reference Atmospheric Model (GRAM) 2010 [15]. We show results from the training process, whose convergence is accelerated through processing of the network features and targets, and we analyze the performance of the LSTM in the reconstruction of simulated density and wind profiles.

\section{Deep neural networks}

In this section, we outline some fundamental concepts in machine learning, general supervised learning algorithms for regression problems, and the RNN and LSTM architectures. The reader is addressed to References [9, 16-19] for an in-depth treatment of these topics.

\section{A. Supervised deep learning for regression problems}

In a regression problem, we assume that there exists a nonlinear mapping $\boldsymbol{F}$ that maps a set of features $\boldsymbol{x} \in \mathbb{R}^{n}$ into a set of targets $\boldsymbol{y} \in \mathbb{R}^{m}$,

$$
\boldsymbol{y}=\boldsymbol{F}(\boldsymbol{x})
$$

The nature of the mapping $\boldsymbol{F}$ is unknown; however, we assume to know $K \gg 1$ samples of feature and target pairs, $\boldsymbol{y}^{(k)}=\boldsymbol{F}\left(\boldsymbol{x}^{(k)}\right), k=1, \ldots, K$. Our aim is to generate an approximate nonlinear mapping $\hat{\boldsymbol{F}}$ that gives an estimate for 
the targets

$$
\hat{\boldsymbol{y}}=\hat{\boldsymbol{F}}(\boldsymbol{x} ; P) \approx \boldsymbol{y},
$$

where, with a slight abuse of notation, we define $P$ as a discrete set of real parameters. The nonlinear mapping must be generated by exploiting the information available through the dataset $C=\left\{\left(\boldsymbol{x}^{(k)}, \boldsymbol{y}^{(k)}\right), k=1, \cdots, K\right\}$. If the mapping $\hat{\boldsymbol{F}}$ closely approximates targets corresponding to samples not present in the dataset $C$, it is said to generalize well.

The solution of the regression problem starts by assuming that $\hat{\boldsymbol{F}}$ belongs to a specific family of functions. One of the simplest choices is a shallow feedforward network, in which the estimates $\hat{\boldsymbol{y}}$ are obtained through

$$
\hat{\boldsymbol{y}}=\boldsymbol{\theta}(W \cdot \boldsymbol{x}+\boldsymbol{b})=\hat{\boldsymbol{F}}(\boldsymbol{x} ; W, \boldsymbol{b}),
$$

where $\boldsymbol{\theta}$ is a nonlinear activation function that is applied element-wise to an affine transformation. In eq. (3), the set of parameters is $P=\{W, \boldsymbol{b}\}$, with $W$ and $\boldsymbol{b}$ respectively consisting of a weight matrix and a bias vector. Common choices for the activation function are the hyperbolic tangent, the sigmoid

$$
\theta(x)=\frac{1}{1+e^{-x}},
$$

or the rectified linear unit (ReLU),

$$
\theta(x)=\max (0, x)
$$

It is often useful to assume expressions for $\hat{\boldsymbol{F}}$ that are more involved than eq. (3). Deep neural networks prescribe the analytical form of the nonlinear mapping as a sequential composition of activation functions and affine transformations. In a deep feedforward network with $L+2$ layers, the outputs are computed through

$$
\begin{aligned}
& \hat{\boldsymbol{y}} \stackrel{\text { def }}{=} \boldsymbol{o}^{(L)}=\boldsymbol{\theta}\left(\boldsymbol{i}^{(L)}\right) \\
& \boldsymbol{i}^{(L)}=W^{(L-1)} \cdot \boldsymbol{o}^{(L-1)}+\boldsymbol{b}^{(L-1)} \\
& \boldsymbol{o}^{(L-1)}=\boldsymbol{\theta}\left(\boldsymbol{i}^{(L-1)}\right) \\
& \vdots \\
& \boldsymbol{o}^{(l)}=\boldsymbol{\theta}\left(\boldsymbol{i}^{(l)}\right) \\
& \vdots \\
& \boldsymbol{o}^{(1)}=\boldsymbol{\theta}\left(\boldsymbol{i}^{(1)}\right) \\
& \boldsymbol{i}^{(1)}=W^{(0)} \cdot \boldsymbol{o}^{(0)}+\boldsymbol{b}^{(0)} \\
& \boldsymbol{o}^{(0)} \stackrel{\text { def }}{=} \boldsymbol{x}
\end{aligned}
$$


where $P=\left\{W^{(l)}, \boldsymbol{b}^{(l)} ; l=0, \cdots, L-1\right\}$. The first and last layer are the input and output layers, respectively, and the remaining $L$ are called the hidden layers. Each of the layers is composed by a number of units, which is equal to the number of rows of the weight matrices $W^{(l)}$ and to the dimension of the bias vectors $\boldsymbol{b}^{(l)}$. Deep neural networks are easily described through directed acyclic graphs, where the nodes correspond to the activation functions and the edges to the affine transformations.

In supervised learning, we seek the set of parameters $P$ minimizing a cost or loss function $\mathcal{L}(\boldsymbol{y}, \hat{\boldsymbol{y}}, P)$, which is a measure of the distance between the estimate $\hat{\boldsymbol{y}}$ and the true value of the feature $\boldsymbol{y}$. A common choice for regression problems is the mean squared error

$$
\mathcal{L}=\frac{1}{2 K^{\prime}} \sum_{k=1}^{K^{\prime}}\left\|\hat{\boldsymbol{y}}\left(\boldsymbol{x}^{(k)} ; P\right)-\boldsymbol{y}^{(k)}\right\|^{2}
$$

where $1 \leq K^{\prime} \leq K$. This is a computationally expensive numerical optimization problem that is solved through some form of the iterative gradient-descent algorithm. For instance, given the value of a parameter $p \in P$ (an element of a weight matrix or a bias vector), the gradient descent algorithm updates its value at each iteration as

$$
p \leftarrow p-\alpha \frac{\partial \mathcal{L}}{\partial p},
$$

where $\alpha$ is called the learning rate, and the gradient $\partial \mathcal{L} / \partial p$ for the hidden layers is computed through the backpropagation algorithm [9]. The choice of $K^{\prime}$ is determined as a trade-off between gradient accuracy and computational effort. Rather than using the entire training set in for the calculation of the gradient $\left(K^{\prime}=K\right)$, it is often convenient to use only a subset, or mini-batch of $K^{\prime}<K$ samples, which results in a noisy approximation of the total gradient. Mini-batch training often converges to flat minima of the loss function, which improves generalization capability of the network [20]. The algorithm for $K^{\prime}<K$ is called stochastic gradient descent, since the $K^{\prime}$ samples used in the calculation of the gradient at each iteration are chosen at random. A training epoch corresponds to a pass of the gradient descent algorithm through each sample of the training set, and an iteration corresponds to a pass through one mini-batch.

The total set of $K$ samples is split into three subsets called the training, validation, and test sets. Samples from the training set are used in the calculation of the gradients, while those from the validation set are used to assess the performance of the network on samples that have not been encountered before, i.e. its generalization capability. Usually, the training is stopped when the loss computed on the validation set is stationary. The test set is used to assess the performance of the network independently from the training and validation sets, ensuring that the network does not overfit to the validation set.

Deep feedforward networks of the form in eq. (4) are able to approximate a wide class of functions arbitrarily well, provided that the number of parameters is sufficiently large and that the training converges [10]. Intuitively, the larger are the number of units for each layer (i.e. the network width) and the number of layers (the network depth), the greater 
the computational power or capacity of the network.

In the context of this work, the nonlinear mapping $\boldsymbol{F}$ consists of the implicit relation between trajectories followed by a dynamical system and the parameters of such system. This is an inverse problem, in which the parameters of the dynamical system must be estimated from the trajectories.

\section{B. Recurrent Neural Networks}

Recurrent Neural Networks are a class of deep neural networks that is particularly efficient in performing predictions from sequences of arbitrary length $n$,

$$
\boldsymbol{\Xi}=\left(x_{1}, x_{2}, \cdots, x_{i}, \cdots, x_{n-1}, x_{n}\right)
$$

where $\boldsymbol{x}_{i}$ is the feature vector at the $i$ th step. Here and in the following, we will assume that steps along the sequence corresponds to steps in time. RNNs represent information from the time sequence in eq. (7) through the hidden state $\boldsymbol{h}$. After assigning an initial value $\boldsymbol{h}_{0}$, the hidden state $\boldsymbol{h}_{i}$ and the output of the neural network $\boldsymbol{o}_{i}$ at the $i$ th step along the sequence is computed through the forward propagation equations

$$
\begin{aligned}
\boldsymbol{h}_{i} & =\boldsymbol{\theta}\left(\boldsymbol{i}_{i}\right) \\
\boldsymbol{i}_{i} & =W \cdot \boldsymbol{h}_{i-1}+U \cdot \boldsymbol{x}_{i}+\boldsymbol{b}
\end{aligned} \quad i \geq 1 .
$$

The hidden state is a lossy representation of the information from all past sequence elements. Information is lost because the dimension of the hidden state is fixed while the sequence length $n$ can increase indefinitely, therefore it is possible to store only part of the information content of the sequence of feature vectors $\boldsymbol{\Xi}$. In particular, the information stored in $\boldsymbol{h}$ corresponds to the task-relevant information content of the sequence, i.e. the information that leads to the minimization of the loss. The hidden state is the mechanism through which information from past steps flows forward, and informs the RNN predictions. In a sequence-to-sequence RNN architecture, the outputs of the network,

$$
\boldsymbol{o}_{i}=V \cdot \boldsymbol{h}_{i}+\boldsymbol{c}, \quad i=1, \cdots, n,
$$

are computed at each step of the sequence. In contrast, in a sequence-to-one RNN architecture the outputs are computed only at the final step of the sequence, $i=n$. The parameters of the network do not depend on the step $i$ in eq. (8); i.e. the parameters are shared across time steps. Sharing parameters across time steps enables the generalization of the network to sequence lengths not present in the training set, and greatly decreases the computational cost of the training. Similarly to deep feedforward network, RNNs are universal in the sense that they can approximate arbitrarily well a wide class of functions, given that the training converges and that the network is composed by enough units [11]. 


\section{RNN training and vanishing gradients}

Training of a RNN is analogous to that of a deep feedforward network. In particular, we seek the parameter values that minimize a prescribed loss function which, in the case of a sequence-to-one RNN, can be expressed through eq. (5).

The gradient of the loss with respect to the parameters is computed by backpropagation through time (BPTT) [16] When stepping back in time in BPTT, the magnitude of the gradient is proportional to the high-order derivatives of the activation function, whose magnitude tends to vanish with increasing order. Therefore, the gradient of the loss also tends to vanish the longer we step back in time, which prevents the RNN from learning long-term dependencies of the outputs from past steps in the sequence. Several approaches have been devised to keep the magnitude of the gradient from vanishing and solve the problem of long-term dependencies, for instance by introducing skip connections across several time steps or through teacher forcing [9]. One of the most effective approaches consists in substituting the hidden layer in eq. (4) with a deep sub-network, the Long Short-Term Memory cell.

\section{Long Short-Term Memory Networks (LSTMs) for sequence-to-one predictions}

The LSTM cell solves the problem of long-term dependencies by introducing a control mechanisms that preserves the gradient magnitude across long sequences. As for the classical RNN, the LSTM cell output at the $i$ th timestep $\boldsymbol{o}_{i}$ depends on the current input $\boldsymbol{i}_{i}$ and the past output $\boldsymbol{o}_{i-1}$. The calculation of the LSTM cell output starts by computing the outputs $\boldsymbol{g}_{i}, \boldsymbol{f}_{i}, \boldsymbol{u}_{i}$ of the input, forget, and output gates, with each gate corresponding to a network layer,

$$
\begin{aligned}
& \boldsymbol{g}_{i}=\boldsymbol{\theta}\left(U \cdot \boldsymbol{x}_{i}+V \cdot \boldsymbol{o}_{i-1}+\boldsymbol{b}\right) \\
& \boldsymbol{f}_{i}=\boldsymbol{\theta}\left(U^{\prime} \cdot \boldsymbol{x}_{i}+V^{\prime} \cdot \boldsymbol{o}_{i-1}+\boldsymbol{b}^{\prime}\right) \\
& \boldsymbol{u}_{i}=\boldsymbol{\theta}\left(U^{\prime \prime} \cdot \boldsymbol{x}_{i}+V^{\prime \prime} \cdot \boldsymbol{o}_{i-1}+\boldsymbol{b}^{\prime \prime}\right) .
\end{aligned}
$$

The cell state $s_{i}$, which is analogous to the hidden state in the classical RNN, is

$$
\boldsymbol{s}_{i}=\boldsymbol{g}_{i} \odot \tanh \left(U^{\prime \prime \prime} \cdot \boldsymbol{x}_{i}+V^{\prime \prime \prime} \cdot \boldsymbol{o}_{i-1}+\boldsymbol{b}^{\prime \prime \prime}\right)+f_{i} \odot s_{i-1},
$$

where $\odot$ denotes element-wise multiplication. Finally, the cell output $\boldsymbol{o}_{i}$ is computed through multiplication of the output layer by the nonlinear transformation of the cell state,

$$
\boldsymbol{o}_{i}=\boldsymbol{u}_{i} \odot \tanh \left(s_{i}\right) .
$$

The parameters in the weight matrices and bias vectors are found during training through BPTT, analogously to the classical RNN. We refer to the recent work by Sherstinsky for an exhaustive treatise of the LSTM architecture, including the training equations [19]. 


\section{Generation of trajectory and measurements dataset}

As to generate sequences of states and of measurements for the training of the network, we simulate 10000 entry trajectories, each encountering different density and winds obtained from the Mars GRAM 2010 atmospheric model [15].

For each of the trajectories in the dataset, we draw the GRAM parameters $\tau_{\text {dust }}, A_{0}, S$ from uniform distributions,

$$
\begin{aligned}
\tau_{\text {dust }} & \sim \mathcal{U}(0.1,3) \\
\log _{10} A_{0} & \sim \mathcal{U}\left(-1, \log _{10} 8\right) \\
\log _{10} S & \sim \mathcal{U}\left(\log _{10} 40,4\right),
\end{aligned}
$$

where $\tau_{\text {dust }}$ is the background dust optical depth, and $A_{0}, S$ are respectively the mean value and the scale parameter of the longitude-dependent density wave modeled in GRAM [15]. The scale parameter $S$ is expressed in kilometers. Although GRAM can simulate correlated stochastic perturbations in density and wind, we only consider their deterministic components of density and wind in this work.

\section{A. FNPEG guidance}

The Fully Numerical Predictor-corrector Entry Guidance (FNPEG) algorithm is a bank-angle only entry guidance method originally proposed by Lu [21]. The method considers the dynamics of an unpowered entry vehicle in a reference frame rotating with the planet, which are described through six equations of motion in spherical coordinates, ${ }^{*}$

$$
\begin{gathered}
\dot{r}=v \sin \gamma \\
\dot{\theta}=\frac{v \cos \gamma \sin \psi}{r \cos \phi} \\
\dot{\phi}=\frac{v \cos \gamma \cos \psi}{r} \\
\dot{v}=-D-\frac{\mu \sin \gamma}{r^{2}}+\Omega^{2} r \cos \phi(\sin \gamma \cos \phi-\cos \gamma \sin \phi \cos \psi) \\
\dot{\gamma}=\frac{1}{v}\left[L \cos \sigma+\left(\frac{v^{2}}{r}-\frac{\mu}{r^{2}}\right) \cos \gamma+2 \Omega v \cos \phi \sin \psi+\Omega^{2} r \cos \phi(\cos \gamma \cos \phi+\sin \gamma \cos \psi \sin \phi)\right] \\
\dot{\psi}=\frac{1}{v}\left[\frac{L \sin \sigma}{\cos \gamma}+\frac{v^{2}}{r} \cos \gamma \sin \psi \tan \phi-2 \Omega v(\tan \gamma \cos \psi \cos \phi-\sin \phi)+\frac{\Omega^{2} r}{\cos \gamma} \sin \psi \sin \phi \cos \phi\right],
\end{gathered}
$$

*Equations (18) to (23) have been derived in the absence of wind, thus the velocity of the vehicle in the Mars-fixed reference frame coincides with the velocity with respect to the flow. 
where

$$
\begin{aligned}
D & =-\frac{1}{2} \rho v^{2} \frac{C_{D} A}{m} \\
L & =-\frac{1}{2} \rho v^{2} \frac{C_{L} A}{m}
\end{aligned}
$$

are the lift and drag accelerations.

The aim of the FNPEG guidance is to bring the vehicle from the initial conditions at Entry Interface (EI) to the target range with respect to the landing site $s^{*}$ by controlling the lift components $L \cos \sigma, L \sin \sigma$. The final conditions for the entry are prescribed as the desired final radius and velocity $r^{*}, v^{*}$, which define the final orbital energy $-e\left(r^{*}, v^{*}\right)=-e_{\mathrm{f}}=v^{* 2} / 2-\mu / r^{*}$. This is achieved through a bank angle-only control law in which the bank angle magnitude is parametrized as a linear function of $e$,

$$
|\sigma(e)|=\sigma_{0}+\frac{e-e_{0}}{e_{\mathrm{f}}-e_{0}}\left(\sigma_{\mathrm{f}}-\sigma_{0}\right)
$$

where $\sigma_{\mathrm{f}} \geq 0$ is either a prescribed constant, or is set equal to $\sigma_{0}$ if a constant bank angle profile is desired.

FNPEG separately addresses the problems of longitudinal guidance, which minimizes the final range error, and lateral guidance, which keeps the vehicle on the course to the landing site through bank reversals. In the longitudinal guidance problem, the value of $\sigma_{0}$ is found to minimize the final range error $z\left(\sigma_{0}\right)=s\left(e_{\mathrm{f}} ; \sigma_{0}\right)-s^{*}$. This is accomplished through an adaptive Gauss-Newton method, in which the value of $\sigma_{0}$ at the $(n+1)$ th iteration is

$$
\sigma_{0}^{(n+1)}=\sigma_{0}^{(n)}-\beta^{(n)} \frac{z\left(\sigma_{0}^{(n)}\right)}{\partial z\left(\sigma_{0}^{(n)}\right) / \partial \sigma_{0}},
$$

where $\beta^{(n)}$ is a step-size regulation factor ensuring that $z\left(\sigma_{0}{ }^{(n+1)}\right)<z\left(\sigma_{0}{ }^{(n)}\right)$, and the partial derivative of the range error at $n>2$ is computed through the approximation

$$
\frac{\partial z\left(\sigma_{0}^{(n)}\right)}{\partial \sigma_{0}} \approx \frac{z\left(\sigma_{0}^{(n+1)}\right)-z\left(\sigma_{0}^{(n)}\right)}{\sigma_{0}^{(n+1)}-\sigma_{0}^{(n)}} .
$$

Neglecting the rate of rotation of the planet $\Omega$, the longitudinal equations of motion become

$$
\begin{gathered}
\dot{r}=v \sin \gamma \\
\dot{v}=-D-g \sin \gamma \\
\dot{\gamma}=\frac{1}{v}\left[L \cos \sigma-\left(g-\frac{v^{2}}{r}\right) \cos \gamma\right],
\end{gathered}
$$


Table 1 Initial conditions and time at the Entry Interface (EI) for the trajectories in the dataset.

\begin{tabular}{ccccccc}
\hline$t_{\mathrm{EI}}$ & $h_{\mathrm{EI}}$ & $\theta_{\mathrm{EI}}$ & $\phi_{\mathrm{EI}}$ & $v_{\mathrm{EI}}$ & $\gamma_{\mathrm{EI}}$ & $\psi_{\mathrm{EI}}$ \\
\hline 18 Feb 2021 12:30 UTC & $130 \mathrm{~km}$ & $90^{\circ}$ & $45^{\circ}$ & $4000 \mathrm{~m} \mathrm{~s}^{-1}$ & $-15^{\circ}$ & $70^{\circ}$ \\
\hline
\end{tabular}

Table 2 FNPEG target conditions for the trajectories in the dataset. Specifying $\left(\theta^{*}, \phi^{*}\right)$ given $\left(\theta_{0}, \phi_{0}, \psi_{0}\right)$ is equivalent to assigning $s_{\mathrm{go}}$.

\begin{tabular}{rcc}
\hline$s_{\mathrm{go}}$ & 7.952 & $\circ$ \\
$r_{\mathrm{f}}$ & 3408.2 & $\mathrm{~km}$ \\
$v_{\mathrm{f}}$ & 1214 & $\mathrm{~m} \mathrm{~s}^{-1}$ \\
$e_{\mathrm{f}}$ & 11.8944 & $\mathrm{~km}^{2} \mathrm{~s}^{-2}$ \\
\hline$\theta^{*}$ & 101.031 & $\circ$ \\
$\phi^{*}$ & 47.203 & $\circ$ \\
\hline
\end{tabular}

where $g=\mu / r^{2}$ is the gravitational acceleration, $D=-\rho v^{2} C_{D} A /(2 m)$ is the drag acceleration and $L=-\rho v^{2} C_{L} A /(2 m)$ is the lift acceleration. To make the detection of the overshoot of the landing site possible, the range $s$ is signed, i.e. $s>0$ corresponds to uprange and $s<0$ to downrange from the landing site. The range is computed by numerically integrating

$$
\dot{s}=-\frac{v \cos \gamma}{r}
$$

along with eqs. (29) to (31). Equation (32) is valid in the hypothesis that the offset of the heading angle $\psi$ from the course to the landing site is small. This is accomplished by the lateral guidance, which keeps the vehicle on course to the target by assigning bank reversals whenever the heading offset $\Delta \Psi$ exceeds a velocity-dependent deadband

$$
\Delta \Psi=c_{1} v+c_{0},
$$

where $c_{1}, c_{0}$ are coefficients chosen to minimize the number of bank reversals while preserving sufficient crossrange accuracy.

References [21,22] also propose a control augmentation scheme in order to respect load and heat constraints, and to eliminate phugoid oscillations for mid lift-to-drag vehicles. We do not implement the augmented control law as it is not essential for the scope of this work. 
Table 3 Parameters used for the FNPEG guidance algorithm.

\begin{tabular}{cc}
\hline Time step & $1 \mathrm{~s}$ \\
Bank profile & Constant \\
Initial bank angle guess & $90^{\circ}$ \\
Gauss-Newton tolerance & $10^{-4}$ \\
Numerical integration tolerance & $10^{-8}$ \\
Lateral deadband width at initial velocity $\Delta \Psi_{\mathrm{i}}$ & $2^{\circ}$ \\
Lateral deadband width at final velocity $\Delta \Psi_{\mathrm{f}}$ & $1.5^{\circ}$ \\
\hline
\end{tabular}

\section{B. Reference trajectory design}

The entry vehicle parameters correspond to those of the Hypersonic Inflatable Aerodynamic Decelerator (HIAD) $[23,24]$. HIAD has an initial entry mass of $49 \times 10^{3} \mathrm{~kg}$, a ballistic coefficient of $155 \mathrm{~kg} \mathrm{~m}^{-2}$, and a lift-to-drag ratio $L / D=0.15$. We implemented a closed-loop MATLAB EDL simulation code in which the guidance is provided by the FNPEG algorithm described in the previous section, and the trajectory is computed by integrating the equations of motion in Cartesian coordinates in a Mars-centered reference frame with its first axis pointing towards the intersection of the prime meridian with the equator, third axis pointing towards Mars' pole of rotation, and second axis completing the right-handed frame. The equations of motion are

$$
\begin{gathered}
\dot{\boldsymbol{r}}=\boldsymbol{v} \\
\dot{\boldsymbol{v}}=-\frac{\mu}{r^{3}} \boldsymbol{r}+\boldsymbol{L}+\boldsymbol{D}-2 \boldsymbol{\Omega} \times \boldsymbol{v}-\mathbf{\Omega} \times(\mathbf{\Omega} \times \boldsymbol{r}),
\end{gathered}
$$

where $\boldsymbol{\Omega}=(0,0, \Omega)$ and the lift and drag vectors are obtained through

$$
\begin{aligned}
& \boldsymbol{L}=L\left(\cos \sigma \frac{\boldsymbol{v}_{\infty} \times\left(\boldsymbol{r} \times \boldsymbol{v}_{\infty}\right)}{v_{\infty}\left\|\boldsymbol{r} \times \boldsymbol{v}_{\infty}\right\|}-\sin \sigma \frac{\boldsymbol{r} \times \boldsymbol{v}_{\infty}}{\left\|\boldsymbol{r} \times \boldsymbol{v}_{\infty}\right\|}\right) \\
& \boldsymbol{D}=-D \frac{\boldsymbol{v}_{\infty}}{v_{\infty}}
\end{aligned}
$$

where $\boldsymbol{v}_{\infty}=\boldsymbol{v}-\boldsymbol{w}$ is the velocity with respect to the flow, and the bank angle $\sigma$ is computed by the FNPEG guidance. ${ }^{\dagger}$ We use the same initial and FNPEG target conditions in all the trajectories in the dataset; these are shown in tables 1 and 2 . The target conditions have been obtained by propagating the initial conditions in table 1 ballistically until reaching an altitude of $11 \mathrm{~km}$, with no wind and assuming the density to follow the exponential law

$$
\rho(h)=\left[0.0263 \exp \left(-\frac{h}{10.1536 \mathrm{~km}}\right)\right] \mathrm{kg} \mathrm{m}^{-3},
$$

\footnotetext{
${ }^{\dagger}$ In eqs. (36) to (37) the bank angle is measured from the $\left(\boldsymbol{r}, \boldsymbol{v}_{\infty}\right)$ plane to the lift vector, while in the FNPEG derivation the bank angle is measured from the $(\boldsymbol{r}, \boldsymbol{v})$ plane. Since $v / w \ll 1$, the difference in the definitions of the bank angle does not affect the controlled dynamics significantly.
} 


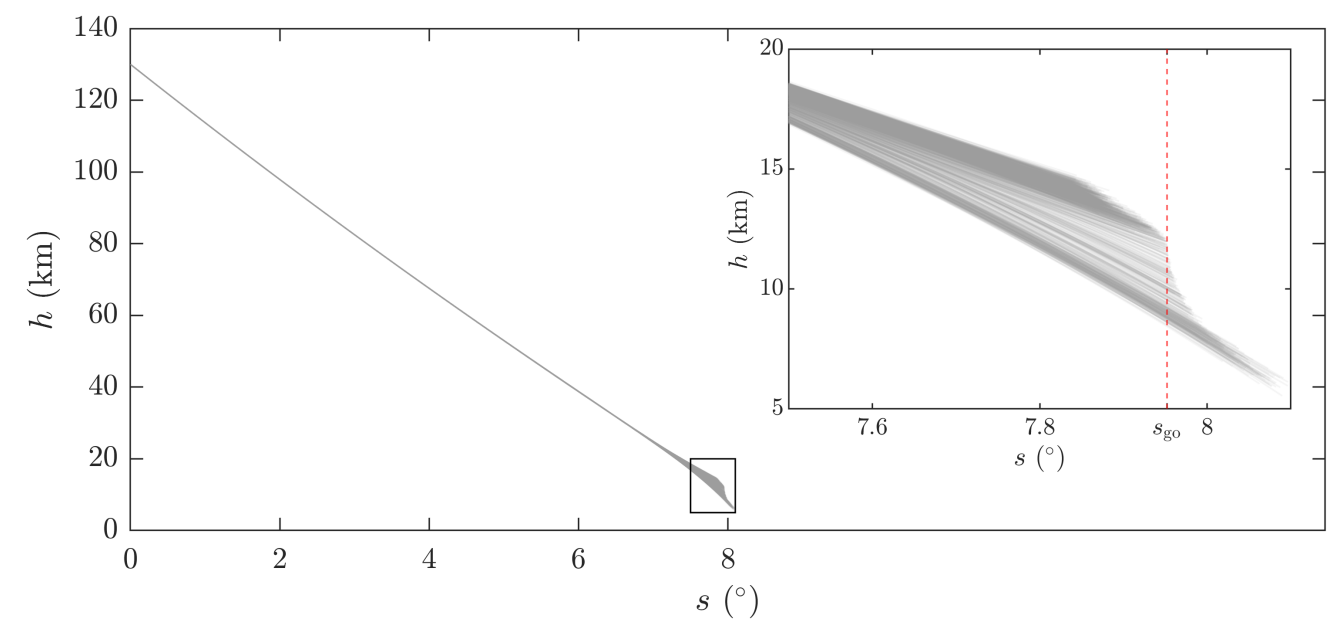

Fig. 1 Altitude as a function of range from the initial conditions for the trajectories in the dataset. The in-panel axes are drawn in correspondence of the black rectangle in the main axes, and show a zoom of the final part of the trajectories. The red dashed line in the secondary axes is drawn at the target range-to-go $s_{\mathrm{go}}$.

which is a least-squares exponential fit for $h \in[0 \mathrm{~km}, 60 \mathrm{~km}]$ to the density profiles $\rho\left(h ; t_{\mathrm{EI}}, \theta_{\mathrm{EI}}, \phi_{\mathrm{EI}}, \tau_{\mathrm{dust}}, A_{0}, S\right)$, where $t_{\mathrm{EI}}, \theta_{\mathrm{EI}}, \phi_{\mathrm{EI}}$ correspond to the values in table 1 . The final energy and range-to-go $-e_{\mathrm{f}}, s_{\mathrm{go}}$ are taken as those attained at $h=11 \mathrm{~km}$ in the ballistic propagation. The FNPEG parameters, shown in table 3, are also the same for all trajectories. In each simulation, the FNPEG entry guidance starts when the acceleration experienced by the spacecraft crosses a threshold of $1.471 \mathrm{~m} \mathrm{~s}^{-2}$, corresponding to 0.15 Earth g's. In the lifting phase prior to the start of entry guidance, the vehicle flies with a trim bank angle of $90^{\circ}$.

\section{Analysis of FNPEG performance and atmospheric quantities}

All of the trajectories in the dataset correspond to direct entries in which the altitude decreases monotonically, as it can be inferred from the altitude as a function of range in fig. 1, and are terminated at the final energy value $e_{\mathrm{f}}$ in table 2 . Since the FNPEG guidance assumes the exponential density law in eq. (38) and no wind, the final range reached in each of the trajectories is considerably different from $s_{\mathrm{go}}$. Each trajectory is affected by different density and wind along the descent, thus the power dissipated along each of them is different. Trajectories with a lower average power dissipation end with a higher kinetic energy and thus with lower potential energy. A small number of trajectories significantly overshoot the target range, which is caused by a lack of control authority for particularly low average densities and power dissipation rates.

We show the density encountered by each of the trajectories as a function of range in the left panel of Figure 2 shows, and the East and North components of the wind velocity $\boldsymbol{w}=\left(w_{E}, w_{N}\right)$ along with its magnitude $w$ are shown in the right panel. ${ }^{+}$The density increases with range as progressively lower altitudes are crossed. The right panel of fig. 2 shows that the behavior of the wind velocity is not monotonous and not trivial to predict, with significant shearing.

\footnotetext{
${ }^{\ddagger}$ Due to the hypothesis of hydrostatic equilibrium [25], Mars GRAM 2010 does not predict any vertical wind component.
} 

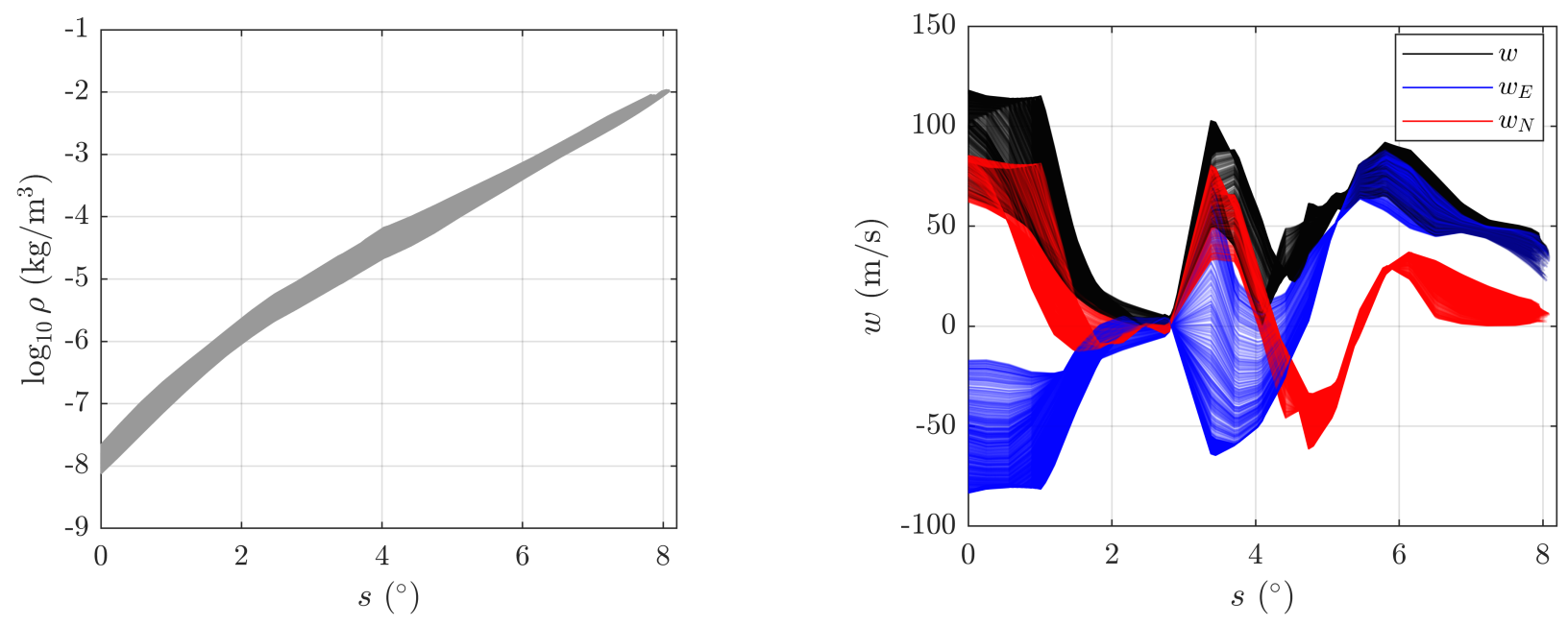

Fig. 2 Density and wind velocity experienced by the trajectories in the dataset as a function of the range from the initial conditions $s$. In the right panel, we show the East and North wind velocity components $w_{E}, w_{N}$, along with the wind velocity norm $w=\sqrt{w_{E}^{2}+w_{N}^{2}}$.

There are considerable dispersions in the density and wind profiles experienced by the trajectories with the choice of sampling for the GRAM parameters in eqs. (15) to (17).

\section{Preparation of network targets}

For each trajectory in the dataset, we record the history of the density and wind velocity experienced along the descent. Since the altitude is a strictly decreasing function of time $(\mathrm{d} h / \mathrm{d} t<0$ always) we can consider it as the independent variable, and the functions $t(h), \theta(h), \phi(h)$ are well defined. With this change of variables, the density $\rho$ and wind velocity $\boldsymbol{w}=\left(w_{E}, w_{N}\right)$ are functions of the altitude exclusively,

$$
\rho=\rho(h, t(h), \theta(h), \phi(h)), \quad \boldsymbol{w}=\boldsymbol{w}(h, t(h), \theta(h), \phi(h)) .
$$

We design the network architecture to predict the East and North wind components $w_{E}\left(h_{j}\right), w_{N}\left(h_{j}\right)$, along with the quantities

$$
\eta\left(h_{j}\right)=\sqrt{B-\log _{10} \rho\left(h_{j}\right)},
$$

which we denominate pseudo-densities. The quantity $B$ in eq. (40) ensures that the argument of the square root is positive. We will consider $B=0$ in the following, since $-\log _{10} \rho$ is positive for all altitudes if the units of $\rho \operatorname{are~} \mathrm{kg} \mathrm{m}^{-3}$. The prediction altitudes $h_{j}$ range in the interval $\left[h_{\min }, h_{\max }\right]$, discretized as

$$
h_{j}=h_{\min }+(j-1) \Delta h,
$$




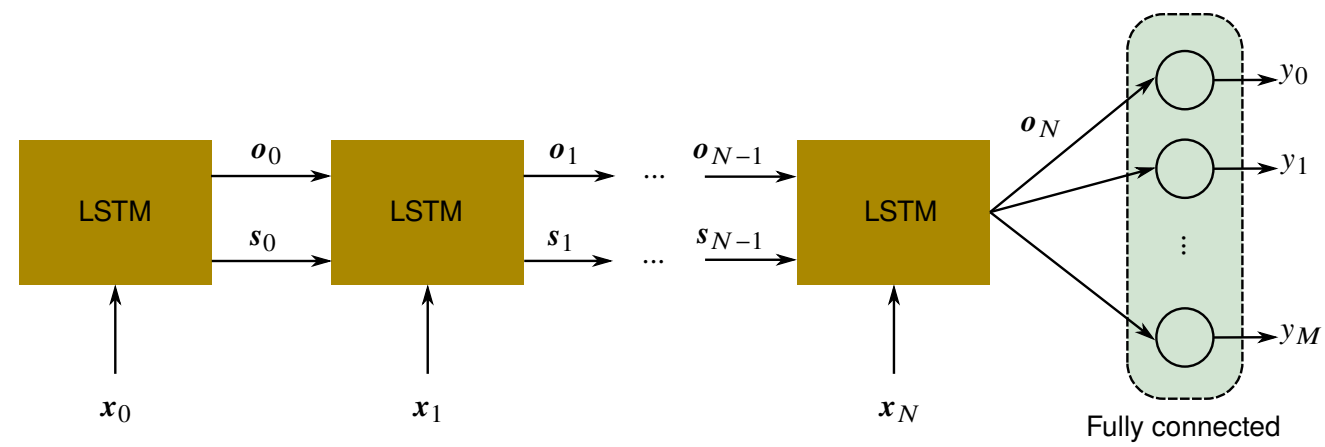

Fig. 3 Computational graph of the LSTM network architecture.

where $h_{\min }=5 \mathrm{~km}, h_{\max }=79 \mathrm{~km}$, and $\Delta h=2 \mathrm{~km} .{ }^{\S}$

Since the equations of motion in each simulation are integrated using time as the independent variable, we linearly interpolate the pseudo-density and wind components experienced by each trajectory in the interval $\left[h_{\min }, h_{\max }\right]$ to obtain $\eta\left(h_{j}\right), w_{E}\left(h_{j}\right), w_{N}\left(h_{j}\right)$ in the discrete set of prediction altitudes $h_{\min }, h_{2}, \cdots, h_{j}, \cdots, h_{\max }$. If a trajectory terminates at an altitude $h_{\mathrm{f}}$ higher than $h_{\min }$, the density and wind velocity for all altitudes $h_{j}<h_{\mathrm{f}}$ are linearly extrapolated.

\section{LSTM architecture and training}

The LSTM network architecture used in this work is portrayed in fig. 3, and it predicts pseudo-density and wind velocity from sequences of feature vectors $\boldsymbol{\Xi}=\left(\boldsymbol{x}_{1}, \boldsymbol{x}_{2}, \cdots\right)$. The dataset consists of 10000 samples corresponding to the trajectories simulated with the closed-loop code, with each sample composed by a feature vector sequence and a target vector.

Each feature vector consists of the state in spherical coordinates and measured acceleration components in a Mars-fixed frame, besides the bank angle value at the past timestep. To obtain the state in spherical coordinates at each timestep, we propagate the controlled dynamics by integration of eqs. (34) to (35). The radius vector and velocity magnitudes, longitude, and latitude are obtained as

$$
\begin{aligned}
r & =\|\boldsymbol{r}\| \\
v & =\|\boldsymbol{v}\| \\
\theta & =\operatorname{atan} 2\left(r_{y}, r_{x}\right) \\
\phi & =\operatorname{asin}\left(\frac{r_{z}}{r}\right)
\end{aligned}
$$

where $r_{x}, r_{y}, r_{z}$ are the position components in the Mars-fixed frame. To obtain the flight-path and heading angles, we

\footnotetext{
${ }^{\S}$ Knowledge of density in this altitude range is particularly important for EDL systems performance [3].
} 
first compute the velocity vector components in the East, North, Radial frame,

$$
\left(\begin{array}{c}
v_{E} \\
v_{N} \\
v_{R}
\end{array}\right)=\left(\begin{array}{ccc}
-\sin \theta & \cos \theta & 0 \\
-\sin \phi \cos \theta & -\sin \phi \sin \theta & \cos \phi \\
\cos \phi \cos \theta & \cos \phi \sin \theta & \sin \phi
\end{array}\right)\left(\begin{array}{c}
v_{x} \\
v_{y} \\
v_{z}
\end{array}\right)
$$

from which we readily obtain the angles as

$$
\begin{aligned}
& \gamma=\operatorname{asin}\left(\frac{v_{R}}{v}\right) \\
& \psi=\operatorname{atan} 2\left(v_{E}, v_{N}\right) .
\end{aligned}
$$

The measured acceleration is the acceleration that would be measured by a sensor attached to the center of gravity of the vehicle, and is equal to the resultant of the aerodynamic accelerations, $\boldsymbol{a}=\boldsymbol{L}+\boldsymbol{D}$. Denoting the $i$ th timestep with the subscript $i$, the $i$ th feature vector is thus

$$
\boldsymbol{x}_{i}=\left(\tilde{r}_{i}, \tilde{\theta}_{i}, \tilde{\phi}_{i}, \tilde{v}_{i}, \tilde{\gamma}_{i}, \tilde{\psi}_{i}, \tilde{a}_{x_{i}}, \tilde{a}_{y_{i}}, \tilde{a}_{z_{i}}, \tilde{\sigma}_{i-1}\right)
$$

where the tilde denotes quantities normalized with the procedure detailed in the following section. Note that the trajectories in the dataset are terminated when reaching the final energy $e_{\mathrm{f}}$, which happens at different final times. Therefore the length of the feature sequence changes for different samples in the dataset.

The dimension of the LSTM cell output at the final timestep $\boldsymbol{o}_{N}$ is equal to the number of hidden LSTM units, i.e. the number of rows in the weight matrices in eqs. (10) to (12). To allow for the number of hidden units to be independent from the number of targets, the final output is passed through a fully connected layer that transforms it into the target vector

$$
\boldsymbol{y}=\left(\tilde{\boldsymbol{\eta}}, \tilde{\boldsymbol{w}}_{E}, \tilde{\boldsymbol{w}}_{N}\right)
$$

The implementation of the LSTM network is carried out with the MATLAB R2020a Deep Learning Toolbox.

\section{A. Feature and target processing}

In order to improve the training convergence, it is helpful to normalize the sets of features and targets by transforming them into sets with zero mean and unitary standard deviation [20]. Let the unnormalized feature vector for the $k$ th sample in the dataset at the $i$ th timestep be

$$
\boldsymbol{\xi}_{i}^{(k)}=\left({ }^{1} \xi_{i}^{(k)},{ }^{2} \xi_{i}^{(k)}, \ldots\right)
$$


where the notation ${ }^{j} \xi_{i}^{(k)}$ denotes the $j$ th component of $\xi_{i}^{(k)}$; for instance, ${ }^{1} \xi_{i}^{(k)}=r^{(k)}\left(t_{i}\right),{ }^{2} \xi_{i}^{(k)}=\theta^{(k)}\left(t_{i}\right)$ are the radius and longitude at the $i$ th timestep of the $k$ th trajectory. The $j$ th element of the normalized feature vector $\boldsymbol{x}_{i}^{(k)}$ is obtained by removing the mean ${ }^{j} \bar{\xi}$ and dividing by the standard deviation ${ }^{j} \breve{\xi}$,

$$
{ }^{j} x_{i}^{(k)}=\frac{{ }^{j} \xi_{i}^{(k)}-{ }^{j} \bar{\xi}}{{ }^{j} \check{\xi}} .
$$

The mean and standard deviation of the $j$ th component are computed over all timesteps (with $N(k)$ being the number of timesteps for the $k$ th trajectory) and samples,

$$
\begin{gathered}
j \bar{\xi}=\frac{1}{K} \sum_{k=1}^{K} \frac{1}{N(k)} \sum_{i=1}^{N(k)}{ }^{j} \xi_{i}^{(k)} \\
j \check{\xi}=\sqrt{\frac{1}{K} \sum_{k=1}^{K} \frac{1}{N(k)} \sum_{i=1}^{N(k)}\left({ }^{j} \xi_{i}^{(k)}-j \bar{\xi}\right)^{2}} .
\end{gathered}
$$

Each element of the target vector is also normalized in a similar fashion,

$$
{ }^{j} y^{(k)}=\frac{{ }^{j} \eta^{(k)}-{ }^{j} \bar{\eta}}{{ }^{j} \check{\eta}}
$$

where ${ }^{j} \bar{\eta},{ }^{j} \check{\eta}$ are the mean and the standard deviation of the $j$ th target component computed over all samples,

$$
\begin{gathered}
{ }^{j} \bar{\eta}=\frac{1}{K} \sum_{k=1}^{K}{ }^{j} \eta^{(k)} \\
{ }^{j} \check{\eta}=\sqrt{\frac{1}{K} \sum_{k=1}^{K}\left({ }^{j} \eta^{(k)}-\bar{\eta}\right)^{2}}
\end{gathered}
$$

\section{B. Network training}

We consider the architecture shown in fig. 3, with a LSTM layer consisting of 256 hidden units. We use $80 \%$ of the samples in the dataset for training, and $10 \%$ each for validation and testing of the network performance. The neural network is trained for a total of 500 epochs with a mini-batch size of 128 samples, corresponding to 31250 iterations. We use the adam optimizer [26] with a constant learn rate of $10^{-3}$ and, to prevent gradient divergence during training, we bound the maximum value of the gradient norm to 1 for each network parameter. The network loss is the average cumulative square error

$$
\mathcal{L}=\frac{1}{K} \sum_{k=1}^{K}\left\|\hat{\boldsymbol{y}}^{(k)}-\boldsymbol{y}^{(k)}\right\|^{2}+\frac{\lambda}{2} \sum_{l=1}^{L} w_{l}^{2},
$$




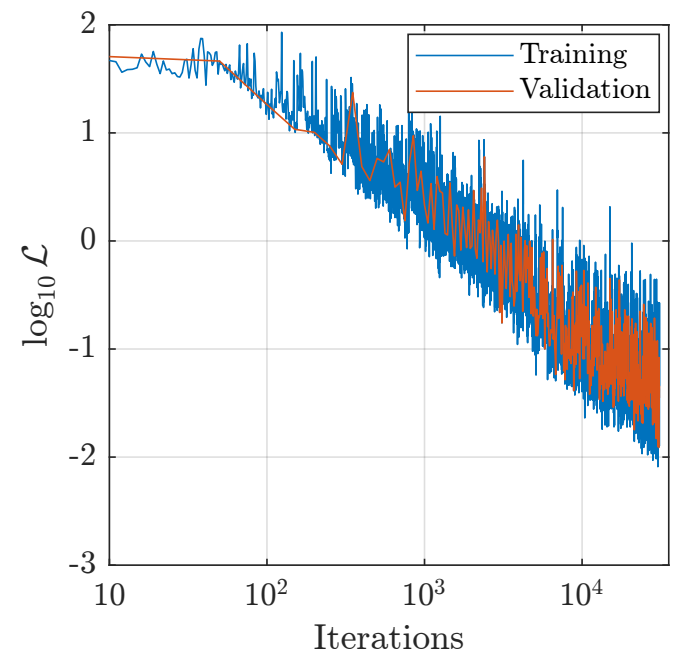

Fig. 4 Network loss computed over the training and validation datasets as a function of iterations.

where $\hat{\boldsymbol{y}}^{(k)}, \boldsymbol{y}^{(k)}$ are the predicted and true target values for the $k$ th sample, $M$ is the dimension of the target vector, and $K$ is either the mini-batch or the validation size depending on whether the loss is evaluated over the training or the validation sets. The second term in eq. (58) is proportional to the squares of the network weights $w_{l}$, with $L$ the total number of weight. This is a regularization term that prevents the training from converging to a sharp minimum, which would result in a poor generalization capability. We set the regularization weight $\lambda=10^{-4}$.

At each iteration, the optimizer updates the values of the network parameters in the direction opposite to the gradient of the loss in eq. (58). Since the loss is computed over a mini-batch, which is a subset of the training set, the resulting loss gradient is an approximation of the gradient computed over the entire training set; effectively, we are introducing noise in the gradient by employing mini-batches. This has generally a positive impact on training because the optimizer will converge to a minimum of the loss function that is more stable, resulting in increased generalization capability [27].

Figure 4 shows the training and validation losses as a function of training iterations. After the first epoch (corresponding to the first 63 iterations), both the training and validation losses decrease by about $20 \mathrm{~dB}$ per decade. Noise in both loss curves due to the mini-batch averaging is apparent. The final training and validation losses are of the same order of magnitude, proving that the trained network is able to generalize well to the validation set. We also considered extending the training to more than 500 epochs; however, the loss stops decreasing once reaching a value of about 0.03 . Decreasing the loss further may be possible by either increasing the number of hidden units or by removing the regularization term in eq. (58); however, this comes at the risk of overfitting to the training set. From the analysis in the following section we found the network performance to be satisfactory, therefore we do not try to decrease the loss further. 


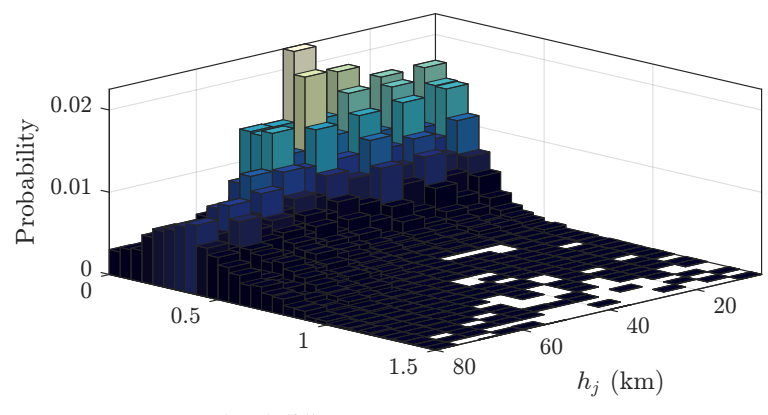

$|\Delta \rho|(\%)$

(a) Relative density error

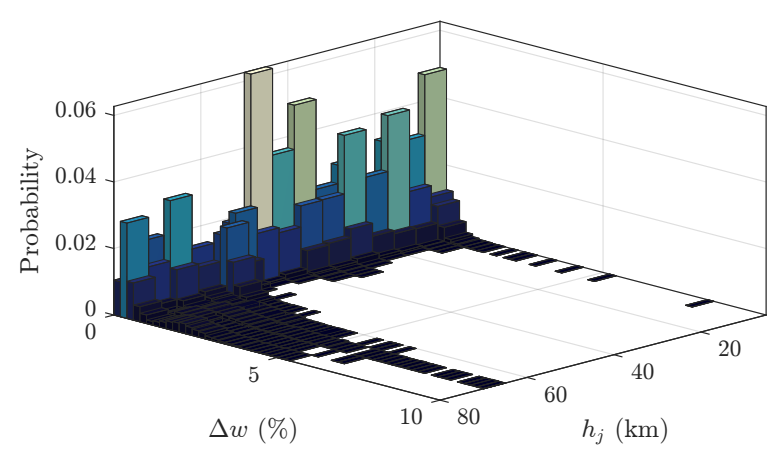

(b) Relative wind error

Fig. 5 Probability histograms of the relative density and wind errors defined through eqs. (62) and (63), as a function of altitude. All errors are evaluated on samples in the testing set. Some outliers with errors in density and wind up to $2 \%$ and $25 \%$ (respectively) are excluded.

\section{Reconstruction of density and wind}

It is difficult to have a direct indication of the performance of the network in predicting density and wind through the final value of the loss in eq. (58), because the target vector $\hat{y}$ is a concatenation of normalized values of pseudo-density and wind as a function of altitude. Moreover, the network performance must be evaluated rigorously on a testing set consisting of trajectories that are not present in either the training or the validation sets. Here, we analyze the performance of the network in predicting the density and wind profile from feature sequences.

For each feature sequence, the LSTM architecture predicts the normalized pseudo-densities $\tilde{\eta}\left(h_{j}\right)$ and wind components $\tilde{w}_{E}, \tilde{w}_{N}$. Deriving the corresponding density and wind is straightforward:

$$
\begin{gathered}
{ }^{j} \eta={ }^{j} \tilde{\eta} \cdot{ }^{j} \check{\eta}+{ }^{j} \bar{\eta} \\
\hat{\rho}\left(h_{j}\right)=10^{-\left({ }^{j} \eta\right)^{2}} \\
\hat{w}_{E}\left(h_{j}\right)={ }^{j} \tilde{w}_{E} \cdot \check{w}_{E}+\bar{w}_{E} \\
\hat{w}_{N}\left(h_{j}\right)={ }^{j} \tilde{w}_{N} \cdot \check{w}_{N}+\bar{w}_{N} .
\end{gathered}
$$

Once the predicted density and wind are computed, an indication of the performance of the network as a function of the prediction altitude can be gained from the relative density and wind errors,

$$
\begin{aligned}
\Delta \rho\left(h_{j}\right) & =100 \frac{\hat{\rho}\left(h_{j}\right)-\rho\left(h_{j}\right)}{\rho\left(h_{j}\right)} \\
\Delta w\left(h_{j}\right) & =100 \frac{\left\|\hat{\boldsymbol{w}}\left(h_{j}\right)-\boldsymbol{w}\left(h_{j}\right)\right\|}{w\left(h_{j}\right)},
\end{aligned}
$$




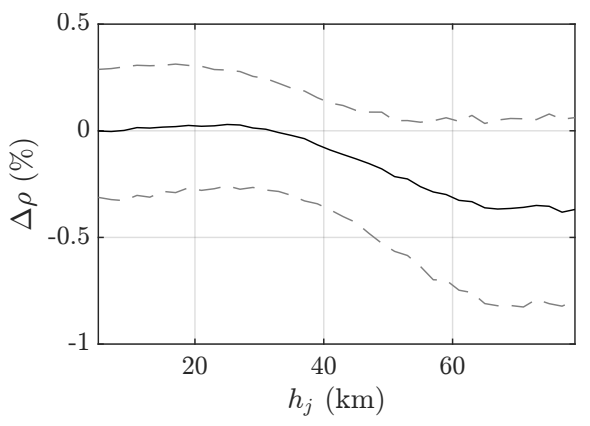

(a) Relative density error

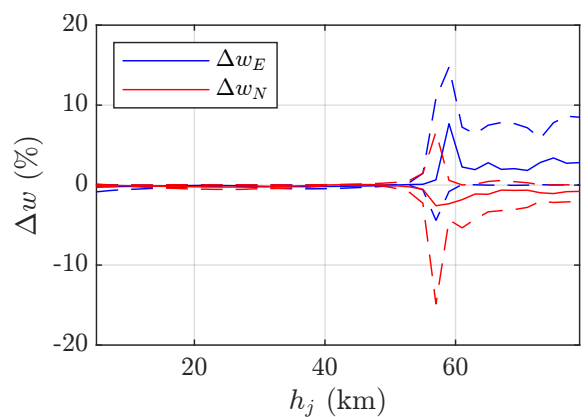

(b) Relative wind error

Fig. 6 Relative density and wind errors as a function of altitude averaged over the samples in the testing set (continuous lines), along with $95 \%$ confidence bounds (dashed lines).

where $\rho\left(h_{j}\right), \boldsymbol{w}\left(h_{j}\right)$ are the true density and wind velocity at altitude $h_{j} \in\left[h_{\min }, h_{\max }\right]$. The distribution of the relative errors as a function of the predicted altitude $h_{j}$ for samples in the testing set is shown in fig. 5. The average accuracy of the density predictions is greater than that of the wind. This is because the LSTM architecture is trained by using information on the state, acceleration, and guidance commands, which are all affected primarily by the density. In particular, the wind error distribution has long tails for altitudes greater than $60 \mathrm{~km}$, where the dynamic pressure encountered by the vehicle is very low and thus the trajectories are not sensitive to changes in wind. In fig. 6, we display the mean and $95 \%$ confidence bounds of the relative density and wind error (the latter evaluated separately on each wind component) as a function of altitude. The average density error is of up to $0.5 \%$, with a slight underestimation bias for $h_{j}>40 \mathrm{~km}$, while the average wind error is less than $2 \%$ in magnitude for $h_{j}<50 \mathrm{~km}$. However, the decreased sensitivity of the trajectory to wind causes errors of up to $15 \%$ (with $95 \%$ confidence) at high altitudes.

In order to gain a synthetic assessment of the network performance independently from the altitude of the predicted atmospheric quantities, we consider the root-mean-squares (RMS) of the relative density and wind errors over altitude,

$$
\begin{aligned}
\Delta \rho_{\mathrm{RMS}} & =\sqrt{\frac{1}{M} \sum_{j=1}^{M} \Delta \rho^{2}\left(h_{j}\right)}, \\
\Delta w_{\mathrm{RMS}} & =\sqrt{\frac{1}{M} \sum_{j=1}^{M} \Delta w^{2}\left(h_{j}\right)},
\end{aligned}
$$

where $M=38$ is the number of altitudes $h_{j}$ in the target vector. We compute the root-mean-square errors separately over the training, validation, and testing sets and present their probability histograms in fig. 7. The statistics of the errors are very similar across the three sets, which proves that the network achieves good generalization capability. In the testing set, the LSTM architecture reconstructs the density with mean RMS and $95^{\text {th }}$-percentile errors of $0.27 \%$ and $0.54 \%$, and the wind with mean RMS and $95^{\text {th }}$-percentile errors of $0.6 \%$ and $1.9 \%$, respectively. 


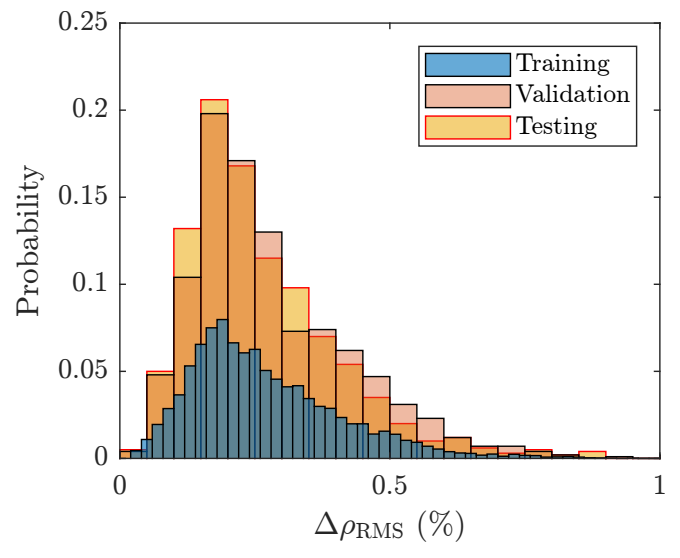

(a) RMS density error

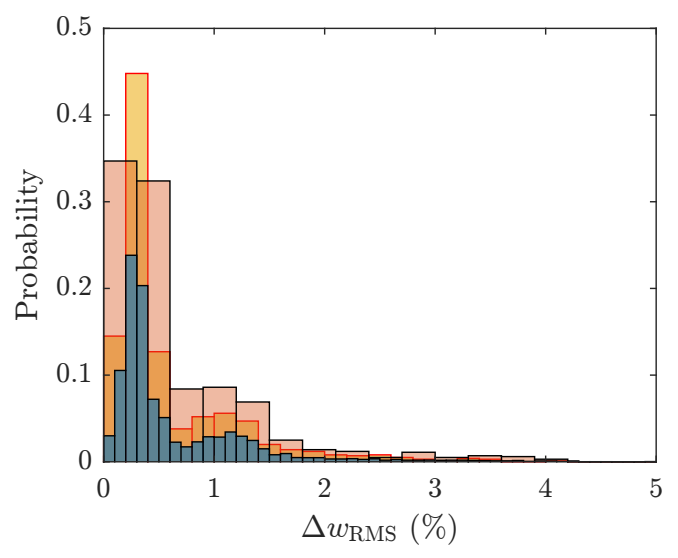

(b) RMS wind error

Fig. 7 Probability histograms of relative RMS density and wind errors over the training, validation, and testing sets. All errors are evaluated on the testing set. We excluded some outliers with RMS errors up to $2 \%$ in density, and up to $8 \%$ in wind.

\section{Discussion and extension of results}

The results in sections IV and V prove that the LSTM architecture reliably reconstructs the density and wind velocity encountered during entry, and is able to generalize well the density and wind predictions to trajectories that are not present in the training and validation sets. In practice, the trained LSTM approximates well the mapping from entry trajectories to encountered densities and wind velocities. The quality of the approximation does not depend on the particular atmospheric model used to generate the dataset; therefore, the predictions from the LSTM architecture should also generalize to trajectories simulated with models different than GRAM 2010, or to state estimates and measurements from actual missions. Within the limits of the present study, we cannot make rigorous claims regarding the generalizability of the proposed method to measurements from actual missions. However, this should be possible by expanding the dataset to include atmospheric profiles measured by recent missions [28] and more realistic simulated trajectories and sensor models [29]. For the latter case, the trajectory and measurement dataset could also be expanded by including dispersions in the initial conditions.

Figure 1 shows that approximating the atmospheric density with a single exponential profile and neglecting winds significantly impacts the accuracy of the entry guidance, with final range errors up to $0.16^{\circ}$ or $9 \mathrm{~km}$. Therefore, an interesting application of the method consists of employing the density and wind predictions to improve the accuracy of the numerical predictor-corrector guidance. In this context, the training process (and possibly the network architecture) must be modified to minimize the loss not only with feature sequences covering the entire entry, but also with partial sequences terminating at intermediate times. The LSTM architecture would continuously update estimates of the density and wind velocity to be expected at future points along the descent from sequences of past features, in a way similar to a nonlinear filter. Such an approach should be compared to state of the art methods for the online estimation of the atmospheric density profile [30,31] or of scaling factors for the aerodynamic forces [32]. The LSTM 
architecture could also be extended to update estimates of the aerodynamic coefficients, and of thrust characteristics during powered descent. In addition, the accuracy of the prediction may benefit by including measurements of air data (such as free-stream static and dynamic pressure) in the features, which would also enable the separation of aerodynamic and atmospheric uncertainties. We expect to pursue the application to the enhancement of entry and powered descent guidance in forthcoming work. Considerations on the reliability of such an enhanced guidance scheme at mid to high Technology Readiness Levels are outside of the scope of our work. However, we note that it would be useful to provide a probabilistic confidence measure associated with atmospheric predictions, e.g. to satisfy design assurance criteria [33]. One could then envision switching to a conventional atmospheric estimation method if the confidence on the atmospheric predictions is too low.

As outlined in section III, the dataset consists of direct entry trajectories exclusively, thus $\mathrm{d} h / \mathrm{d} t<0$ always and we are able to choose altitude (rather than time) as the independent variable for the predicted density and wind velocity. This is not possible for skip entry trajectories in which $\mathrm{d} h / \mathrm{d} t=0$ at some point, and another parametrization must be chosen for the atmospheric quantities. In this regard, choosing energy as the independent variable may be advantageous, especially for applications to the enhancement of onboard guidance.

\section{Conclusions}

We devise a deep learning method based on a LSTM network architecture for the reconstruction of atmospheric density and wind velocity experienced by a vehicle entering the Martian atmosphere from information on its entry trajectory. In particular, the method estimates the profiles of density and wind as a function of altitude from sequences of vehicle states, measured accelerations, and bank angle commands.

We generate a dataset of $10^{4}$ entry trajectories controlled through the FNPEG bank angle law. Each trajectory corresponds to density and wind profiles generated by randomly sampling three parameters of the Mars GRAM 2010 atmospheric model. The trajectories are used to generate feature sequences and target vectors for the training and testing of the network. We prove that the LSTM network generalizes well to samples in the validation set, since the training and validation losses are of the same order of magnitude.

The LSTM network performance is assessed by analyzing relative density and wind errors in the testing dataset. The analysis highlights excellent accuracy in the reconstruction of atmospheric quantities in the testing dataset. In particular, the $95^{\text {th }}$-percentile RMS density and wind errors are $0.54 \%$ and $1.9 \%$, respectively. However, average wind errors may be significantly larger than these values at altitudes of more than $50 \mathrm{~km}$ due to the decreased sensitivity of the trajectories in regions where the aerodynamic forces exerted are particularly low. Finally, we discuss the extension of the results to skip-entry trajectories and the application of the algorithm to actual mission data and online guidance. 


\section{Acknowledgments}

DA thanks Evan Roelke (University of Colorado Boulder) for providing information on the HIAD vehicle, and Roberto Furfaro (The University of Arizona) for having introduced him to neural networks.

\section{Funding Sources}

This work was carried out under the NASA ECF grant 80NSSC18K1510 on "Robust Entry and Landing Guidance Under Dynamic Uncertainty".

\section{References}

[1] Spencer, D. A., Blanchard, R. C., Braun, R. D., Kallemeyn, P. H., and Thurman, S. W., "Mars Pathfinder Entry, Descent, and Landing Reconstruction,” Journal of Spacecraft and Rockets, Vol. 36, No. 3, 1999, pp. 357-366. https://doi.org/10.2514/2.3478, URL https://doi.org/10.2514/2.3478.

[2] Tolson, R., Bemis, E., Hough, S., Zaleski, K., Keating, G., Shidner, J., Brown, S., Brickler, A., Scher, M., and Thomas, P., "Atmospheric Modeling Using Accelerometer Data During Mars Reconnaissance Orbiter Aerobraking Operations," Journal of Spacecraft and Rockets, Vol. 45, No. 3, 2008, pp. 511-518. https://doi.org/10.2514/1.34301, URL https: //arc.aiaa.org/doi/10.2514/1.34301.

[3] Desai, P. N., “All Recent Mars Landers Have Landed Downrange - Are Mars Atmosphere Models Mis-Predicting Density?” LPI Contributions, Lunar and Planetary Institute, Williamsburg, VA, USA, 2008.

[4] Dutta, S., Braun, R. D., Russell, R. P., Striepe, S. A., and Clark, I. G., "Comparison of Statistical Estimation Techniques for Mars Entry, Descent, and Landing Reconstruction,” Journal of Spacecraft and Rockets, Vol. 50, No. 6, 2013 , pp. $1207-1221$. https://doi.org/10.2514/1.A32459, URL https://arc.aiaa.org/doi/10.2514/1.A32459.

[5] Dutta, S., and Braun, R., "Mars Entry, Descent, and Landing Trajectory and Atmosphere Reconstruction," 48th AIAA Aerospace Sciences Meeting, American Institute of Aeronautics and Astronautics, Orlando, Florida, 2010. https://doi.org/10.2514/6.20101210, URL http://arc.aiaa.org/doi/10.2514/6.2010-1210.

[6] Chen, A., Cianciolo, A., Vasavada, A. R., Karlgaard, C., Barnes, J., Cantor, B., Kass, D., Rafkin, S., and Tyler, D., "Reconstruction of Atmospheric Properties from Mars Science Laboratory Entry, Descent, and Landing," Journal of Spacecraft and Rockets, Vol. 51, No. 4, 2014, pp. 1062-1075. https://doi.org/10.2514/1.A32708, URL https://doi.org/10.2514/1.A32708.

[7] Golombek, M. P., Grant, J. A., Parker, T. J., Kass, D. M., Crisp, J. A., Squyres, S. W., Haldemann, A. F. C., Adler, M., Lee, W. J., Bridges, N. T., Arvidson, R. E., Carr, M. H., Kirk, R. L., Knocke, P. C., Roncoli, R. B., Weitz, C. M., Schofield, J. T., Zurek, R. W., Christensen, P. R., Fergason, R. L., Anderson, F. S., and Rice, J. W., "Selection of the Mars Exploration Rover landing sites," Journal of Geophysical Research: Planets, Vol. 108, No. E12, 2003. https://doi.org/10.1029/2003JE002074, URL https://agupubs.onlinelibrary.wiley.com/doi/abs/10.1029/2003JE002074. 
[8] Arridge, S., Maass, P., Öktem, O., and Schönlieb, C.-B., "Solving inverse problems using data-driven models," Acta Numerica, Vol. 28, 2019, pp. 1-174. https://doi.org/10.1017/S0962492919000059, URL https://www.cambridge.org/core/journals/actanumerica/article/solving-inverse-problems-using-datadriven-models/CE5B3725869AEAF46E04874115B0AB15.

[9] Goodfellow, I., Bengio, Y., and Courville, A., Deep Learning, Adaptive computation and machine learning, MIT Press, Cambridge, Massachusetts, USA, 2016. URL http://www.deeplearningbook.org/.

[10] Hornik, K., Stinchcombe, M., and White, H., "Multilayer feedforward networks are universal approximators," Neural Networks, Vol. 2, No. 5, 1989, pp. 359-366. https://doi.org/10.1016/0893-6080(89)90020-8, URL http://www.sciencedirect.com/science/ article/pii/0893608089900208.

[11] Schäfer, A. M., and Zimmermann, H. G., "Recurrent Neural Networks Are Universal Approximators," Artificial Neural Networks ICANN 2006, edited by S. D. Kollias, A. Stafylopatis, W. Duch, and E. Oja, Springer, Berlin, Heidelberg, 2006, pp. 632-640. https://doi.org/10.1007/11840817_66.

[12] Wagner, J., Wilhite, A., Stanley, D., and Powell, R., “An Adaptive Real Time Atmospheric Prediction Algorithm for Entry Vehicles," 3rd AIAA Atmospheric Space Environments Conference, American Institute of Aeronautics and Astronautics, Honolulu, Hawaii, 2011. https://doi.org/10.2514/6.2011-3200, URL http://arc.aiaa.org/doi/10.2514/6.2011-3200.

[13] Wilkinson, M. C., and Meade, A. J., "Neural-Network-Inspired Machine Learning for Autonomous Lunar Targeting," Journal of Aerospace Information Systems, Vol. 11, No. 7, 2014, pp. 458-466. https://doi.org/10.2514/1.I010166, URL https://arc.aiaa.org/doi/10.2514/1.I010166.

[14] Hochreiter, S., and Schmidhuber, J., "Long Short-Term Memory,” Neural Computation, Vol. 9, No. 8, 1997, pp. 1735-1780. https://doi.org/10.1162/neco.1997.9.8.1735, URL https://doi.org/10.1162/neco.1997.9.8.1735.

[15] Justh, H. L., “Mars Global Reference Atmospheric Model 2010 Version: Users Guide,” Tech. Rep. TM-2014-217499, NASA Marshall Spaceflight Center, Huntsville, AL, USA, 2014.

[16] Graves, A., Supervised Sequence Labelling with Recurrent Neural Networks, Studies in Computational Intelligence, Vol. 385, Springer Berlin Heidelberg, Berlin, Heidelberg, 2012. https://doi.org/10.1007/978-3-642-24797-2, URL http://link.springer. com/10.1007/978-3-642-24797-2.

[17] Alpaydin, E., Introduction to machine learning, $3^{\text {rd }}$ ed., Adaptive computation and machine learning, The MIT Press, Cambridge, Massachusetts, 2014.

[18] Google, "Machine Learning Crash Course,”, 2020. URL https://developers.google.com/machine-learning/crash-course.

[19] Sherstinsky, A., "Fundamentals of Recurrent Neural Network (RNN) and Long Short-Term Memory (LSTM) network," Physica D: Nonlinear Phenomena, Vol. 404, 2020. https://doi.org/10.1016/j.physd.2019.132306, URL http://www.sciencedirect.com/ science/article/pii/S0167278919305974, article 132306. 
[20] Montavon, G., Orr, G. B., and Müller, K.-R. (eds.), Neural Networks: Tricks of the Trade, $2^{\text {nd }}$ ed., Lecture Notes in Computer Science, Vol. 7700, Springer Berlin Heidelberg, Berlin, Heidelberg, 2012. https://doi.org/10.1007/978-3-642-35289-8, URL http://link.springer.com/10.1007/978-3-642-35289-8.

[21] Lu, P., "Entry Guidance: A Unified Method," Journal of Guidance, Control, and Dynamics, Vol. 37, No. 3, 2014. https://doi.org/10.2514/1.62605.

[22] Lu, P., Brunner, C. W., Stachowiak, S. J., Mendeck, G., Tigges, M. A., and Cerimele, C. J., "Verification of a Fully Numerical Entry Guidance Algorithm," Journal of Guidance, Control, and Dynamics, Vol. 40, No. 2, 2017. https://doi.org/10.2514/1.G000327.

[23] Dwyer Cianciolo, A. M., and Polsgrove, T., "Human Mars Entry, Descent, and Landing Architecture Study: Phase 2 Summary," American Institute of Aeronautics and Astronautics, 2018. https://doi.org/10.2514/6.2018-5190, URL https: //arc.aiaa.org/doi/abs/10.2514/6.2018-5190.

[24] Bose, D. M., Shidner, J., Winski, R., Zumwalt, C., Cheatwood, F. M., and Hughes, S. J., “The Hypersonic Inflatable Aerodynamic Decelerator (HIAD) Mission Applications Study," AIAA Aerodynamic Decelerator Systems (ADS) Conference, American Institute of Aeronautics and Astronautics, Daytona Beach, Florida, 2013. https://doi.org/10.2514/6.2013-1389, URL http://arc.aiaa.org/doi/10.2514/6.2013-1389.

[25] Haberle, R. M., The atmosphere and climate of Mars, Cambridge University Press, 2017. https://doi.org/10.1017/9781139060172.

[26] Kingma, D. P., and Ba, J., “Adam: A Method for Stochastic Optimization,” arXiv:1412.6980 [cs], 2017. URL http: //arxiv.org/abs/1412.6980, arXiv: 1412.6980.

[27] Keskar, N. S., Mudigere, D., Nocedal, J., Smelyanskiy, M., and Tang, P. T. P., “On Large-Batch Training for Deep Learning: Generalization Gap and Sharp Minima," arXiv:1609.04836 [cs, math], 2017. URL http://arxiv.org/abs/1609.04836, arXiv: 1609.04836.

[28] Sharaf, O., Amiri, S., AlDhafri, S., Withnell, P., and Brain, D., "Sending hope to Mars," Nature Astronomy, Vol. 4, No. 7, ????, pp. 722-722. https://doi.org/10.1038/s41550-020-1151-y, URL https://www.nature.com/articles/s41550-020-1151-y.

[29] Dwyer-Cianciolo, A. M., Sostaric, R. R., Lugo, R. A., Woffinden, D., Karlgaard, C., and Chen, P.-T., "A Simulation Framework for Precision Landing and Hazard Avoidance Technology Assessments," AIAA Scitech 2020 Forum, American Institute of Aeronautics and Astronautics, 2020. https://doi.org/10.2514/6.2020-0366, URL https://arc.aiaa.org/doi/abs/10.2514/6.20200366 .

[30] Masciarelli, J., Rousseau, S., Fraysse, H., and Perot, E., “An analytic aerocapture guidance algorithm for the Mars Sample Return Orbiter," Atmospheric Flight Mechanics Conference, American Institute of Aeronautics and Astronautics, Denver, CO, USA, 2000. https://doi.org/10.2514/6.2000-4116, URL http://arc.aiaa.org/doi/10.2514/6.2000-4116.

[31] Perot, E., and Rousseau, S., "Importance of an On-board Estimation of the Density Scale Height for Various Aerocapture Guidance Algorithms," AIAA/AAS Astrodynamics Specialist Conference and Exhibit, American Institute of Aeronautics and Astronautics, Monterey, California, 2002. https://doi.org/10.2514/6.2002-4734, URL http://arc.aiaa.org/doi/10.2514/6.2002-4734. 
[32] Johnson, B. J., Rocca-Bejar, D., Lu, P., Nikaido, B., Hays, Z. B., D’Souza, S., and Sostaric, R. R., "Pterodactyl: Development and Performance of Guidance Algorithms for a Mechanically Deployed Entry Vehicle," AIAA Scitech 2020 Forum, American Institute of Aeronautics and Astronautics, Orlando, Florida, USA, 2020. https://doi.org/10.2514/6.2020-1011, URL https: //arc.aiaa.org/doi/abs/10.2514/6.2020-1011.

[33] Cluzeau, J. M., Henriquel, X., Rebender, G., Soudain, G., van Dijk, L., Gronskiy, A., Haber, D., Perret-Gentil, C., and Polak, R., "Concepts of Design Assurance for Neural Networks (CoDANN)," Tech. rep., European Union Aviation Safety Agency, 2020. URL https://www.easa.europa.eu/document-library/general-publications/concepts-design-assurance-neural-networks-codann. 